\title{
Response of atmospheric ground level temperature to changes in the total solar irradiance
}

\author{
A.D. Erlykin ${ }^{* a b}$, A.W. Wolfendale ${ }^{b}$ \\ ${ }^{a}$ P.N. Lebedev Physical Institute \\ Leninsky prospect 53, Moscow 119991, Russia \\ ${ }^{b}$ Physics Department, Durham University \\ South Road, Science Laboratories, Durham, DH1 3LE, UK
}

\begin{abstract}
An examination is made of the effect of the total solar irradiance (TSI) on the mean Global surface temperature.

We conclude that TSI changes related to the mean Global temperature are not significant from 1880 onwards but that there is an increase in the sensitivity of temperature to TSI in time.
\end{abstract}

The 34th International Cosmic Ray Conference

30 July - 6 August, 2015

The Hague, The Netherlands

${ }^{*}$ Speaker. 


\section{Introduction}

${ }^{1}$ The role of changes in the TSI in the Climate Change debate is finite but uncertain. Indeed in the latest IPCC Report [1] a range 0.05 to $0.23 \mathrm{Wm}^{-2}$ is quoted, i.e. a range of a factor of 4.6. Further study is clearly needed.

In what follows we examine the relationship between mean Global surface temperature and TSI ( defined as $\alpha$ in units of $K\left(W^{-2}\right)^{-1}$ for individual 11-year Solar Cycles, latitude variations and length of time over which the relationship is sought.

\section{Solar Cycle variations as a function of latitude}

The $\alpha$-values have been determined for Solar Cycles 12-23 and for the usual latitude bands: $90^{\circ} \mathrm{N}-64^{\circ} \mathrm{N}, 64^{\circ} \mathrm{N}-44^{\circ} \mathrm{N}, 44^{\circ} \mathrm{N}-24^{\circ} \mathrm{N}, 24^{\circ} \mathrm{N}-0^{\circ} \mathrm{N}$ and similarly for the South. The values are small ( typically $0.2 \mathrm{~K}\left(\mathrm{Wm}^{-2}\right)^{-1}$ and are mostly characterized by a slow increase with Cycle number until 20 (i.e.1970), followed by a slow fall. Interestingly, this coincides with the peak in the TSI, time series. It is also where the density of $\mathrm{CO}_{2}$ in the atmosphere starts to increase significantly.

The crucial role of the oceans is apparent by the fact that as the 'ocean fraction' of the latitude range considered increases, the change of $\alpha$ with increasing Cycle number, $\mathrm{N}, d \alpha / d N$ falls, at least for Cycles 12-20. Thus, the ocean is having a stabilising effect on atmospheric change. For higher values of $\mathrm{N}$ the situation is reversed, in the sense that $d \alpha / d N$ rises with increasing ocean fraction. In both cases, $d \alpha / d n$ goes to zero as the ocean fraction tends to $100 \%$.

\section{The magnitude of the Global temperature rise due to TSI increases}

From 1880 to 1970 , the TSI increases by $\sim 0,5 \mathrm{Wm}^{-2}$ [2] so that for $\alpha=0.2 \mathrm{~K}\left(\mathrm{Wm}^{-2}\right)^{-1}$ (see §2) the TSI-caused increase in mean Global temperature is $\sim 0.1 K$. This is not negligible in comparison with the observed mean Global temperature rise in this period of $\sim 0.4 K$ [1]. From 1970 onwards, however, where the mean Global temperature continues to rise by a further $1.2^{\circ} \mathrm{K}$ the mean TSI falls by $\sim 0.5 \mathrm{KWm}^{-2}$, a fall largely attributable to the rise in $\mathrm{CO}_{2}$. Using the plt of mean atmospheric $\mathrm{CO}_{2}$ density versus time ( $300 \mathrm{ppm}$ in 1880, $328 \mathrm{ppm}$ in 1970 and $362 \mathrm{ppm}$ in 2010 ) [3] and assuming linearity of TSI and CO-2 with mean Global temperature the whole of the T-rise can be attributed to $\mathrm{CO}_{2}$ rise, the estimated contribution from TSI from 1880 to 1970 being actually $-0.25^{\circ} \mathrm{C}$.

\section{The time dependence of $\alpha$}

An analysis has been made of the effective value of $\alpha$ for different periods of time $(\tau)$, i.e. the extent to which there is a non-linear dependence of temperature changes on TSI changes.Such a dependence might be expected if inertial effects are present.

We have examined the dependence of $\alpha$ on $\tau$ from $\sim 3 \cdot 10^{-3}$ y (i.e. day/night ) via the $11-\mathrm{y}$ variation [4] to $\sim 10^{4}-10^{4} \mathrm{y}$ for the last Ice Age [5] and orbital changes [6].

\footnotetext{
${ }^{1 *}$ Corresponding author: tel +441913343580

E-mail address: a..w.wolfendale@durham.ac.uk
} 
The values of $\alpha$ are, sequentially, approximately $3 \cdot 10^{-3}, 10^{-1}$ and $3 \cdot 10^{-1}\left(\right.$ all in $K\left(\mathrm{Wm}^{-2}\right)^{-1}$ ) .

\section{Conclusions}

The effect of changes of total solar irradiance on the mean Global surface temperature is still somewhat uncertain but it is surely small. Certainly, with respect to the last Century or so there is little relevance to Global Warming. Changes across the surface of the Globe are well correlated with the oceanic fraction and well represented by the thermal inertia associated with the oceans. Such inertial effects are presumably responsible for the slow increase in temperature, TSI correlation with increasing period over which temperatures are considered. Even here, however, TSI effects are probably not large; TSI changes of $\sim 1 \mathrm{Wm}^{-2}$ might be expected and with the actual change in mean surface Global temperature of $\sim 3^{\circ} \mathrm{C}$ the TSI contribution would only be $\sim 10 \%$.

\section{Acknowledgements}

The authors are grateful to the Kohn Foundation for financial support and to the Referees for helpful comments.

\section{References}

[1] IPCC Report Climate change, the physicsl science basis (2014)

[2] TSI: http://lasp.colorado.edu/data/sorce/tsi.data

[3] www.esri.noaa.gov/gmd/ccgg/trends

[4] Lean, J. et al., SORCE contributions to new understanding of global change and solar variability, Solar Phys., 230 (2005) 27

[5] Haigh, J.D., The Sun and the Earth's climate, Living Rev. Solar Phys., 4 (2007) 2

[6] Rusov, V. et al., Private comm. (siiis@t.e.net.ua) (2008) 